\title{
La dinámica controversial de la política científica colombiana 1990-2017: una aproximación sociológica*
}

\author{
Luis Alfonso Chávarro*
}

\footnotetext{
* Este artículo se basa en mi tesis para optar por el título de Doctor en Sociología en la Pontificia Universidad Católica del Perú El malestar con la innovación. Una aproximación sociológica a la dinámica controversial de la politica cientifica colombiana 1990-2017 (2019). Recuperado de http://tesis.pucp. edu.pe/repositorio/handle/123456789/12227

** Licenciado en Literatura y magíster en Sociología por la Universidad del Valle- Cali (Colombia), y doctor en Sociología por la Pontificia Universidad Católica del Perú (PUCP). Actualmente es profesor de la Universidad del Valle en Cali, Colombia. https://orcid.org/0000-0001-5103-6191. Correo electrónico: alfonso.chavarro@correounivalle.edu.co
}

Fecha de recepción: 21/08/2019. Fecha de aceptación: 04/11/2020. 


\title{
La dinámica controversial de la política científica colombiana 1990-2017: una aproximación sociológica
}

\section{RESUMeN}

El presente artículo sintetiza una investigación sobre la apropiación del modelo internacional de innovación en la política científica colombiana y la dinámica controversial generada tras su implementación. El modo de análisis adoptado radica en una integración por combinación de enfoques micro (representaciones y controversias), con enfoques macro (cultura mundial y la teoría de campo), articulados a un procedimiento metodológico en el que se propone como novedad un índice de controversialidad, y tras ello, afloran resultados que muestran cómo la difusión del modelo internacional centrado en la innovación ha sido efectivo, pero que dado su énfasis en la utilidad económica de la ciencia, ha terminado generando controversias entre los agentes científicos, vinculados con el incipiente mundo social de la ciencia en Colombia y evidenciando una percepción de malestar. Ante este descubrimiento, en la discusión se sugiere cómo podría ser un modelo ciudadano alterno de política científica, en donde la representación económica de innovación se equilibre con la innovación social y ambiental.

Palabras clave: política científica, difusión, innovación, controversias, campo científico

\section{The controversial dynamics of Colombian scientific policy 1990-2017: a socio- logical approach}

\begin{abstract}
This article synthesizes an investigation on the appropriation of the international model of innovation in Colombian Scientific Policy and the controversial dynamics generated after its implementation. The mode of analysis adopted lies in an integration by a combination of micro approaches (representations and controversies), with macro approaches (world culture and field theory), articulated to a methodological procedure in which an index of controversiality is proposed as a novelty, And after that, results emerge that show how the dissemination of the international model focused on innovation has been effective, but that given its emphasis on the economic utility of science, it has ended up generating controversies among scientific agents, linked to the incipient social world of science in Colombia, showing a perception of discomfort. Given this discovery, the discussion suggests how an alternative citizen model of scientific policy could be, where the economic representation of innovation is balanced with social and environmental innovation.
\end{abstract}

Keywords: scientific policy, dissemination, innovation, controversies, scientific field. 


\section{INTRODUCCIÓN}

Esta investigación surgió tras descubrir la aparición recurrente de notas y columnas de opinión en la prensa colombiana, en las que se debatía sobre las formas de financiación de la investigación científica. Este hecho condujo a mirar la posibilidad de investigar este fenómeno desde una perspectiva sociológica, pero asumir este reto implicaba introducirse en un campo de investigación poco trabajado por los sociólogos, dado que el tema de las políticas se ha convertido en asunto de administradores y economistas, quienes suelen abordarlo como «evaluación de políticas». Aclarar el camino a seguir implicó, entonces, convertirlo en tema de investigación sociológica, y tras una rica interacción con pares, se planteó aterrizar esta problemática a la luz del interrogante: ¿̨cuáles son las razones de las controversias a que ha lugar dado la política científica colombiana, en qué modelo se inscriben las ideas centrales de dicha política, implementada desde 1990, y cómo dichas ideas centrales y controversias se registran en la percepción de los agentes del mundo social de la ciencia en el país?

El análisis adoptado en la metodología articula dos enfoques micro para asir empíricamente los referentes del problema: el enfoque de las representaciones, para analizar las ideas centrales de la política en cuestión, y el enfoque de las controversias, para el análisis de las reacciones, publicadas en la prensa por actores clave de la comunidad científica colombiana. Y para su comprensión macro, en el marco teórico se articulan dos enfoques más plenamente sociológicos: el enfoque de la cultura mundial, para mirar la difusión internacional de modelos de políticas, y la teoría de campo, para entender el mundo social de la ciencia y sus actores.

\section{LOS MODELOS DE POLÍTICA CIENTÍFICA}

Analizar la política científica implica empezar por mirar sus fundamentos conceptuales y los tipos o modelos implementados, es decir, proporcionar una fundamentación histórica. A grandes rasgos, existe suficiente consenso académico para considerar como política científica la toma de decisiones de los gobiernos sobre la ciencia y su financiación (Albornoz, 2007, p. 47), y su implementación en los distintos países ha estado orientada por la difusión de diversos modelos, como el denominado modelo lineal (Mitcham y Briggle, 2007, p. 143), y otros más recientes.

El modelo lineal surge en tiempos de la Segunda Guerra Mundial en Estados Unidos, cuando el científico Vannevar Bush presenta una propuesta al entonces presidente Franklyn Delano Roosevelt denominada Ciencia, la frontera sin fin (Bush, 1999, p. 5). La denominación de modelo lineal a dicha propuesta reside en que se basa en el algoritmo «lineal» $\mathrm{y}$ «secuencial» de que la investigación en ciencia básica 
es la que permite potenciar la innovación tecnológica, mediante descubrimientos que dan lugar a innovaciones radicales (Mitcham y Briggle, 2007, p. 157). Para ello, los científicos debían ser bien remunerados y disfrutar de autonomía, lo que los alejaría de las tentaciones de intereses económicos o empresariales. Sobre las críticas a dicho modelo surge un segundo modelo, según el cual no siempre es la investigación básica la que empuja a la innovación y su aplicabilidad tecnológica, ya que la innovación puede llegar también desde otros actores y lugares, como los empresarios emprendedores o los usuarios empíricos, entre otros (Rosenberg, 1994, p. 11), lo que llevaría a inferir que el modelo lineal, que privilegia la investigación básica, no necesariamente siempre es el que genera la innovación (Edgerton, 2004, p. 10).

Estos cuestionamientos han conducido a otros autores a manifestar que el modelo lineal de política científica ha privilegiado el rol de las universidades en la generación de conocimiento, pero que, con las nuevas demandas de la sociedad y la economía, han aparecido otros contextos y actores sociales - no siempre vinculados con las universidades-, que han potenciado la innovación, y provienen del sector privado y empresarial o pertenecen a otras redes de actores. Este nuevo modelo ha sido vinculado con lo que se ha denominado el Modo 2 de la ciencia (Gibbons et al., 1994, p. 14), asociado a los cambios en la naturaleza misma de la ciencia y su función. A ello se agrega el papel clave del Estado en la creación de condiciones reales para la investigación básica y aplicada, por lo que algunos han denominado a esta confluencia de actores de la investigación científica el modelo de la triple hélice (Etzkowitz y Leydesdorff, 2000, p. 109). Como se ha podido ver, la política científica tiene dos modelos en pugna: el modelo lineal, que favorece la situación de los científicos al privilegiar su autonomía, y un modelo de mercado, que favorece los intereses de los empresarios y el sector privado en la investigación aplicada (Mitcham y Briggle, 2007, p. 145).

En cuanto a las controversias sobre la política científica, los precedentes se centran en las reacciones a la implementación de estos modelos, desde diversos sectores. Respecto de la implementación del modelo lineal, la iniciativa de Vannevar Bush, una vez convertida en política por el gobierno estadounidense, fue cuestionada por un miembro del Parlamento, el senador Harley Kilgore, quien veía con preocupación que los fondos para la investigación quedaran en manos de unos científicos pertenecientes a unas pocas universidades y desligados de las necesidades de la sociedad estadounidense (Sampat, 2007, p. 20). Asimismo, en la década siguiente, muchas revistas científicas fueron eco de acalorados debates sobre la orientación de la política científica, y en ellos, científicos como Michael Polanyi y Desmond Bernal se trenzaron en una controversia para defender orientaciones opuestas: el primero, la autonomía que propiciaba a los científicos el modelo lineal impuesto por Vannevar Bush desde la posguerra y al que defendió con la idea de la república 
de la ciencia (Polanyi, 1962, p. 54), mientras el segundo aludía a la funcionalidad social de la ciencia (Bernal, 1938, p. 15), más allá de las orientaciones bélicas, económicas o meramente académicas.

En el contexto latinoamericano, el auge de la política científica se empieza a dar desde la década de 1960, con la aparición de los consejos de ciencia y tecnología en varios países (Vessuri, 1994, p. 41). Sin embargo, ya en la década de 1950 se habría dado en América Latina un empuje de la ciencia para la modernización, pero dentro de un marco de sustitución de importaciones que privilegiaba la transferencia tecnológica, por lo que la investigación científica habría quedado en un plano menor (Sagasti, 2013, p. 135). No será hasta la década de 1970, bajo la influencia desarrollista de la UNESCO y la CEPAL, cuando se adopten los primeros instrumentos de gestión de política científica en la región (Albornoz, 2009, p. 71). Ya en la década de 1980, tras el ajuste económico impulsado por el Consenso de Washington, se impone en la política científica de la región la idea de los sistemas de innovación y competitividad (Sagasti, 2013, p. 140), que terminan influyendo de manera importante en la generación de nuevas políticas científicas de la década de 1990 en diversos países de América Latina, entre ellos, Colombia.

En cuanto al surgimiento de controversias, este periplo de iniciativas plasmadas en la política científica en América Latina no estuvo exento de debates. La fuente de cuestionamiento más importante provino de argumentos elaborados desde el PLATS por científicos como Jorge Sábato y Máximo Halty, quienes pedían una mayor regulación de la tecnología extranjera, o de Oscar Varsavsky, quien planteaba la necesidad de una ciencia autónoma en la región y, además, cuestionaba el cientificismo (Albornoz, 2009, p. 66). También por Amílcar Herrera, para quien el problema de América Latina era que la ciencia no tenía demanda local (Herrera, 1995, p. 6).

Ya en tiempos de globalización, hacia la década de 1990, se constata en los países de América Latina una tendencia generalizada centrada en la innovación para la productividad y generación de valor agregado. Sin embargo, dicha tendencia no se ha materializado de la misma manera en todos los países, dado que algunos han privilegiado indicadores de acceso, participación y equidad, como Argentina y Brasil, mientras que otros se han orientado más al pie de la letra de las recomendaciones internacionales, como Chile, México y Colombia (Casas, 2013, p. 4).

\subsection{La política científica colombiana}

En la múltiple bibliografía existente sobre política científica colombiana, se ha tratado de periodizar dicha política, llegando a estimarse de tres a cinco períodos. Sin embargo, una revisión sistemática permite a quien aquí escribe delimitar claramente cuatro períodos, como puede verse en el cuadro 1 . 
Cuadro 1. Periodización de la política de ciencia y tecnología en Colombia

\begin{tabular}{|c|c|c|}
\hline Períodos & Descripción & Característica clave \\
\hline $\begin{array}{l}\text { Primer } \\
\text { período } \\
\text { Antes de } \\
1968\end{array}$ & $\begin{array}{l}\text { Creación de institutos de ciencia claves para el } \\
\text { desarrollo del país } \\
\text { En } 1968 \text { se crea COLCIENCIAS. } \\
\text { No hay organización institucional. }\end{array}$ & $\begin{array}{l}\text { Era de los consejos } \\
\text { de investigación en } \\
\text { América Latina. }\end{array}$ \\
\hline $\begin{array}{l}\text { Segundo } \\
\text { período } \\
\text { De } 1968 \text { a } \\
1990\end{array}$ & $\begin{array}{l}\text { En } 1978 \text { se da la primera política científica en } \\
\text { Colombia, dentro del Plan de Desarrollo Plan } \\
\text { de Integración Nacional (PIN). Allí ya aparece la } \\
\text { necesidad de vincular la investigación a elevar la } \\
\text { productividad }\end{array}$ & $\begin{array}{l}\text { Primera política } \\
\text { científica }\end{array}$ \\
\hline $\begin{array}{l}\text { Años } \\
\text { ochenta }\end{array}$ & $\begin{array}{l}\text { En el Plan de Desarrollo siguiente, Cambio con } \\
\text { equidad del ańo 1982, la ciencia se eleva a factor } \\
\text { de desarrollo nacional, por lo que objetivos de ese } \\
\text { plan apuntan a fortalecer las universidades y las } \\
\text { comunidades científicas. } \\
\text { Al inicio del siguiente período de gobierno, en el } \\
\text { Plan de Economía Social, aparece un vacío en lo } \\
\text { relativo a ciencia y tecnología; sin embargo, hacia } \\
\text { 1988, reaparece como asunto de debate público la } \\
\text { necesidad de una política de ciencia y tecnología } \\
\text { en el país, por lo que se celebra un foro de ciencia y } \\
\text { tecnología para el desarrollo. } \\
\text { Hacia } 1989 \text { se decreta el Año de la Ciencia y la } \\
\text { Tecnología en el país, y se crea la Misión de Ciencia } \\
\text { y Tecnología, que presentará sus resultados a } \\
\text { comienzos de la década de } 1990 \text { (DNP, 1990). }\end{array}$ & $\begin{array}{l}\text { Necesidad de vincular } \\
\text { la ciencia al desarrollo } \\
\text { nacional } \\
\text { Misión de ciencia y } \\
\text { tecnología } \\
\text { Cierta organización } \\
\text { institucional }\end{array}$ \\
\hline $\begin{array}{l}\text { Tercer } \\
\text { período } \\
1990 \text { a } 2009\end{array}$ & $\begin{array}{l}\text { Entra en vigencia la Ley } 29 \text { de } 1990 \text {, que recupera } \\
\text { las recomendaciones internacionales de vincular } \\
\text { la investigación a la productividad económica. Se } \\
\text { crea el Sistema Nacional de Ciencia y Tecnología a } \\
\text { comienzos de la década. }\end{array}$ & $\begin{array}{l}\text { Vigencia de la Ley } 29 \\
\text { de } 1990 \\
\text { Cambio estructural } \\
\text { Sistema Nacional de } \\
\text { Ciencia y tecnología } \\
\text { (SNCyT) }\end{array}$ \\
\hline $\begin{array}{l}\text { Cuarto } \\
\text { período } \\
2009 \text { a la } \\
\text { actualidad }\end{array}$ & $\begin{array}{l}\text { Entra en vigencia la Ley } 1286 \text { de } 2009 \text {, centrada en } \\
\text { el imperativo de la innovación. } \\
\text { Sistema Nacional de Ciencia, Tecnología e } \\
\text { Innovación. } \\
\text { Política científica dependiente de política de } \\
\text { competitividad. }\end{array}$ & $\begin{array}{l}\text { Vigencia de la Ley } \\
1286 \text { de } 2009 \\
\text { Sistema Nacional de } \\
\text { Ciencia, Tecnología e } \\
\text { Innovación (SNCTI) } \\
\text { Agenda de } \\
\text { competitividad }\end{array}$ \\
\hline
\end{tabular}

Fuente: Elaboración personal del autor (Chávarro, 2018) con base en las fuentes citadas: Garay et al. (1998), Forero y Villaveces et al. (2002), Jaramillo (2007), Malaver y Vargas (2005), Guzmán (2014), CONPES (2009). 
Como se puede ver, habría dos momentos, anteriores a 1990, caracterizados por una importante formación de recurso humano en investigación: antes de 1968, momento de la creación de COLCIENCIAS (Jaramillo et al., 2007, p. 303), y de 1968 hasta 1988-1989, cuando se da el consenso necesario para una política científica centrada en la articulación con el desarrollo económico (Guzmán, 2014, p. 24). Luego de la década de 1990, habría un tercer período caracterizado por dos momentos claves: primero, con la entrada en vigencia de la Ley 29 de 1990, hasta llegar a la Ley 1286 de 2009, cuando se inicia otro momento en que resulta central la innovación. Estos dos momentos, pertenecientes al período de globalización posterior a 1990, constituyen el objeto de análisis del presente trabajo.

En cuanto a la aparición de controversias sobre la política científica colombiana, las críticas surgidas en la literatura académica y las evaluaciones de dicha política constituyen una primera manifestación del debate. Aunque en algunos trabajos académicos ya se destacaba el impulso de la política científica desde 1990 para buscar el avance de la ciencia y la tecnología en el país como factor de desarrollo e instrumento para crear ventajas competitivas en la globalización, en su valoración de la contribución a la productividad ya estos trabajos señalaban algunas deficiencias observadas con la implementación de la nueva política, como la falta de cobertura del mismo sistema de ciencia y tecnología, la falta de financiación de la investigación, la poca eficacia en el uso de recursos, y otros planteamientos, que se pueden sintetizar bajo la idea de insuficiencia: "[...] se evidencia aún una insuficiencia, tanto en el esquema institucional como en la dotación y asignación de recursos» (Garay et al., 1998, p. 222). En el mismo sentido, en un trabajo de 2011, se admitía que, al iniciar la segunda década del siglo, se observaba un interesante proceso de proyectos montados funcionando y con cierta continuidad, pero, asimismo, se mencionaban falencias con la implementación de la política científica, como el bajo nivel de inversión en CTI - ya que el porcentaje del PIB seguía siendo muy bajo- y se subrayaba la imperiosa necesidad de formación de doctores, ya que, en comparación con los países cercanos, seguía siendo limitada. También se enfatizaba en el bajo número de patentes de residentes y la falta de promoción de lo relativo a la propiedad intelectual (Turriago y Hernández, 2011, p. 59). Posteriormente, en una evaluación realizada por la OCDE a las políticas de innovación en Colombia, al mirar el análisis DOFA allí realizado, entre las debilidades se resaltaba la dependencia del país de las materias primas —especialmente las minerales-, pero también los bajos niveles de productividad, los altos niveles de desigualdad de ingresos y el bajo nivel de la investigación. Entre las amenazas, aparecían la falta de diversificación productiva, los procesos engorrosos para la solicitud de proyectos, la incapacidad para actualizar conocimientos, y el no llevar a cabo programas que atendieran las necesidades sociales (OCDE, 2014, p. 44). 
A estas críticas procedentes de estudios de carácter económico, centradas en la evaluación de la política científica, se sumaron otros basados en indicadores propiamente científicos y de un carácter más crítico respecto de las ideas que son centrales en esta política científica, como la productividad y la competitividad. Estos trabajos confirmaban que en este país, al igual que en otros de América Latina, se ha venido diseñando e implementando una política científica basada en un modelo productivo de innovación, cuyos resultados evidenciaban contradicciones entre objetivos y realizaciones en términos de los mismos indicadores de innovación que aconsejaban utilizar los difusores de dicha política científica. Muchos estudios no reseñados aquí han subrayado esa contradicción entre las metas y discursos, y los deficientes resultados obtenidos. Sin embargo, sobre la misma construcción de los indicadores usados para la evaluación de políticas científicas existía discusión, ya que estos instrumentos suelen ser diseñados para medir y evaluar la producción de los investigadores desde una perspectiva desarrollista, que considera la ciencia como factor de productividad en la economía. En este sentido, tales indicadores serían unos instrumentos de homogeneización del mundo de la ciencia, orientados a permitir la acción de algunos agentes externos al campo científico, como gestores, tecnocracia, políticos y empresarios (Gómez, 2005, p. 242).

Asimismo, tras entrar en vigencia la Ley 1286 de 2009, en otro trabajo sobre la política científica colombiana, se llamaba a cuestionar su idea central porque conducía a «instrumentalizar la razón al servicio de determinados fines» (Hoyos, 1991, p. 417). En el mismo sentido, respecto de esta ley, en otra investigación se afirmaba que, con la política de ciencia en Colombia, lo que se había impuesto era un deber ser para la competitividad (Hernández, 2011, p. 152). Como se puede ver, los cuestionamientos a la política científica implementada desde 1990 indicaban la presencia latente de controversias que más tarde llegarían a medios de opinión, como la prensa.

\section{MarCo de ANÁlisis}

El marco teórico adoptado se basa en una articulación de diversos enfoques: en primer lugar, para la primera afirmación de la hipótesis, consistente en que la política científica colombiana es resultado de la apropiación de un modelo de política internacional centrado en la innovación, aquí se adopta el enfoque de la cultura mundial (Meyer, Boli et al., 1997, p. 145), según el cual las políticas de los países son resultado de la difusión de modelos internacionales y tienden a ser adoptadas de manera mimética, conduciendo a una homogenización e isomorfismo institucional (Di Maggio y Powell, 1999, p. 108). Para su abordaje empírico, se echa mano del enfoque de las representaciones (Bauer y Gaskell, 1999, p. 168) y así poder iden- 
tificar las formas y tipos ideales en que se da el anclaje y objetivación de las ideas difundidas en los modelos internacionales de dicha política.

En segundo lugar, para la segunda parte de la afirmación hipotética, consistente en las reacciones de los agentes del mundo social de la ciencia nacional ante la implementación de la política científica, se acude a la teoría de campo (Bourdieu, 2003, p. 68) para observar cómo la difusión de modelos no cae en el vacío, sino en un campo de fuerzas y de agentes que reclaman más autonomía, controvierten y disienten de muchos lineamientos de tal política. Para su abordaje empírico, se acude al enfoque de las controversias (Brante y Elzinga, 1990), procedente de la sociología de la ciencia, ya que permite mirar cómo los debates y discusiones se hacen desde un lugar dentro del campo o mundo social de la ciencia.

\section{Metodología}

\subsection{Implicaciones de la articulación de enfoques}

Como se puede ver en el cuadro 2, con la articulación de enfoques se hacía necesario poder asir empíricamente el proceso de difusión de un modelo y su apropiación en una política científica nacional, lo cual se hizo a partir del análisis de tres objetos: primero, los textos de la política científica (leyes, planes de desarrollo y documentos CONPES); segundo, las notas de prensa que registran los debates como prueba de la reacción a la implementación de la política científica, y tercero, la calibración de la relación entre lo contenido en los documentos textuales de la política científica y las reacciones registradas en la prensa, pero desde la percepción subjetiva de actores concretos del mundo social de la ciencia. 


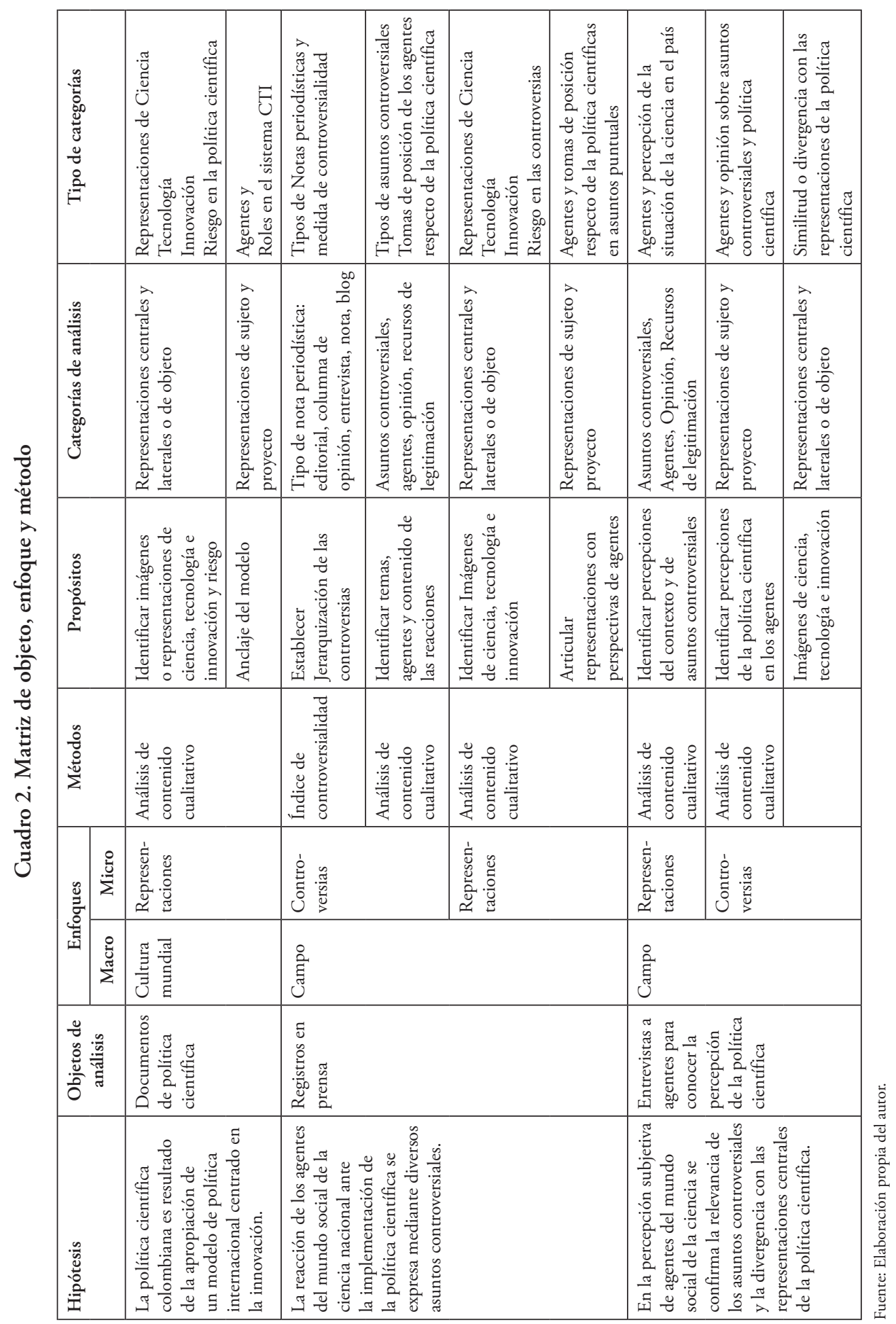




\subsection{Categorías de análisis, guías y muestreo}

\subsubsection{Categorias de representaciones}

La definición de las categorías de representaciones se hizo en términos de la similitud o analogía predominante, o el marco metafórico implicado. La matriz de categorías resultante permitió, entonces, construir tipos ideales a partir de fundamentos conceptuales, y con base en ello se diseñó la ficha a aplicar para analizar las representaciones de ciencia, tecnología, e innovación en los textos de la política científica.

Los tipos ideales de representaciones utilizados como categorías de análisis fueron, en primer lugar, respecto de la ciencia: la ciencia como producto (el conocimiento), la ciencia como regla (método científico), la ciencia como sujeto (instituciones y comunidad científica). En segundo lugar, respecto de la tecnología: la tecnología como artefacto, como ciencia aplicada, como sistema, como transferencia y como construcción social. En tercer lugar, respecto de innovación: las representaciones de innovación tecnológica, innovación económica e innovación competitiva, y las representaciones alternas de innovación (innovación social y ambiental).

\subsubsection{Categorías de asuntos controversiales, muestreo y medición de controversialidad}

Para identificar los asuntos controversiales, las categorías clave utilizadas fueron: tipo de asunto, actores implicados, opinión y recursos de justificación. Para el análisis de prensa se escogieron las notas publicadas en la prensa de referencia en Colombia (periódicos El Tiempo y El Espectador y revista Semana, y eventualmente, otras publicaciones subsidiarias de la casa matriz de cada uno de estos tres medios) por su pertinencia como registro de asuntos controversiales. El muestreo de las notas no obedece a criterios cuantitativos sino cualitativos; es decir, se realiza un muestreo intencional exhaustivo, dado que se toma la mayoría de las notas aparecidas en estos medios como soporte de la veracidad de dichos debates, pero con una irregularidad explicable por la casi inexistente aparición de notas sobre la política científica en la década de 1990, pero que luego de 2009 se vuelve numerosa.

En cuanto a la medida de la controversialidad, aunque se toman como referentes algunos trabajos realizados en Europa para medir la importancia que la prensa otorga a la ciencia (Dimpoulos y Koulaidis, 2002, p. 225), así como la identificación de controversias científicas en la prensa (Díaz Moreno, 2012, p. 54), en el presente trabajo se optó por una medida alternativa de la jerarquía de las noticias, esta vez, con base en la discusión, debate o controversialidad que potencian, por lo que se considera a la variable tipo de noticia como el referente empírico para identificar ese grado de impacto en la opinión. En otras palabras, se parte de la hipótesis de que una noticia sobre la política científica tiene más efecto en la opinión pública si se registra como tema por los columnistas de opinión o si llega a ser el objeto de 
la nota editorial del periódico. A partir de ese supuesto, se ha diseñado un índice de medida de controversialidad de los asuntos de la política científica, en la que, según el tipo de noticia, el asunto recibe una puntuación, siendo la nota editorial la de más puntos (5), seguida de la nota de opinión (4), la de entrevista (3), la noticia corriente (2) y la de blog (1). De esa manera, la puntuación obtenida por el total de noticias sobre el tipo de noticia de cada asunto le otorga una jerarquía de controversialidad.

\subsubsection{Categorías de análisis, guía y muestreo de entrevistas}

Para identificar las percepciones sobre la política científica vigente, se realizaron entrevistas no estructuradas a agentes partícipes del mundo de la ciencia. Antes de ello, se elaboraron categorías de análisis derivadas de tres grandes tipos de categorías de percepción: las percepciones relacionadas con el contexto —es decir, la situación de la ciencia en el país—, las percepciones puntuales de la política científica —en otras palabras, la percepción de la política en sí, la percepción de sus problemas, las apuestas para solucionar dichos problemas y la opinión sobre los asuntos controversiales acerca de la misma política registrados en la prensa, entre otras. Finalmente, las percepciones sobre las ideas centrales y laterales en dicha política, de donde emergen las representaciones.

Para la selección de las personas a entrevistar, se realizó un muestreo intencional con la premisa de que los escogidos tuvieran vinculación con el sistema de ciencia, tecnología e innovación y que representaran de alguna manera a los sectores propios de dicho sistema. En ese sentido, los seleccionados pertenecen al menos a tres de los diferentes sectores del sistema: investigación, gestión y apropiación social. Cuando la selección intencional no se pudo dar, por varias dificultades para el contacto, se recurrió al método de bola de nieve, es decir, algunos de los entrevistados fueron sugeridos por otros anteriormente entrevistados. Algunas de las entrevistas se realizaron directamente y otras se hicieron a distancia por vía de la plataforma Skype. Finalmente, los entrevistados seleccionados son representativos de las tres regiones del país en donde se concentran universidades, investigadores y patentes: Bogotá, Valle del Cauca y Antioquia.

\section{Resultados y CONCLUSIONES}

\subsection{Las representaciones predominantes en los documentos de la política científica colombiana}

El análisis de los documentos de política científica implicó la revisión de múltiples fuentes primarias, cuya cantidad revisada se puede ver en el cuadro siguiente. 


\section{Cuadro 3. Fuentes primarias analizadas}

\begin{tabular}{|c|c|c|}
\hline Fuentes primarias & Fecha & Descripción general \\
\hline Ley 29 & 1990 & $\begin{array}{l}\text { Disposiciones para el fomento de la investigación científica y el } \\
\text { desarrollo tecnológico }\end{array}$ \\
\hline PND La Revolución Pacífica & 1990 & Plan Nacional de Desarrollo 1990-1994 \\
\hline Decreto 393 & 1991 & $\begin{array}{l}\text { Normas sobre asociación para actividades } \\
\text { científico-tecnológicas }\end{array}$ \\
\hline Decreto 585 & 1991 & Creación del Consejo Nacional de Ciencia y Tecnología \\
\hline Decreto 591 & 1991 & $\begin{array}{l}\text { Regulación de modalidades específicas de contratos de } \\
\text { fomento de actividades científico-tecnológicas }\end{array}$ \\
\hline PND El Salto Social & 1994 & Plan Nacional de Desarrollo 1994-1998 \\
\hline Documento Conpes 2739 & 1994 & Política Nacional de Ciencia y Tecnología 1994-1998 \\
\hline Cambio para construir la paz & 1998 & Plan Nacional de Desarrollo 1998-2002 \\
\hline Documento Conpes 3080 & 2000 & Política Nacional de Ciencia y Tecnología 2000-2002 \\
\hline Documento Conpes 3146 & 2001 & $\begin{array}{l}\text { Estrategia para consolidar la ejecución del Plan Nacional para } \\
\text { la Prevención y Atención de Desastres (PNPAD) en el corto y } \\
\text { mediano plazo }\end{array}$ \\
\hline $\begin{array}{l}\text { PND Hacia un Estado } \\
\text { Comunitario }\end{array}$ & 2002 & Plan Nacional de Desarrollo 2002-2006 \\
\hline Documento Conpes 3297 & 2004 & $\begin{array}{l}\text { Agenda interna para la productividad y la competitividad: } \\
\text { metodología }\end{array}$ \\
\hline Conpes Social 0091 & 2005 & $\begin{array}{l}\text { Metas y estrategias de Colombia para el logro de los objetivos } \\
\text { de desarrollo del milenio - } 2015\end{array}$ \\
\hline $\begin{array}{l}\text { Estado Comunitario: } \\
\text { Desarrollo para Todos }\end{array}$ & 2006 & Plan Nacional de Desarrollo 2006-2010 \\
\hline Visión 2019 II Centenario & 2006 & $\begin{array}{l}\text { Fundamentar el crecimiento y el desarrollo social en la ciencia, } \\
\text { la tecnología y la innovación. }\end{array}$ \\
\hline $\begin{array}{l}\text { Plan de Ciencia Tecnología e } \\
\text { Innovación de Bogotá D.C. } \\
2007-2019\end{array}$ & 2007 & Bogotá, sociedad del conocimiento \\
\hline Documento Conpes 3582 & 2008 & Política Nacional de Ciencia, Tecnología e Innovación \\
\hline $\begin{array}{l}\text { Informe Nacional de } \\
\text { Competitividad 2008-2009 }\end{array}$ & 2008 & \\
\hline $\begin{array}{l}\text { Colombia construye y siembra } \\
\text { futuro }\end{array}$ & 2008 & $\begin{array}{l}\text { Política Nacional de Fomento a la Investigación y la } \\
\text { Innovación de Colciencias }\end{array}$ \\
\hline Ley 1286 & 2009 & Modificación de la Ley 29 de 1990 \\
\hline PND Prosperidad para Todos & 2010 & Plan Nacional de Desarrollo 2010-2014 \\
\hline PND Todos por un Nuevo Pais & 2014 & Plan Nacional de Desarrollo 2010-2014 \\
\hline $\begin{array}{l}\text { Gran Pacto } \\
\text { MedellInnovation }\end{array}$ & 2014 & $\begin{array}{l}\text { Se plantea trabajar por una sociedad y economía basada en } \\
\text { conocimiento, equidad, inclusión y responsabilidad social }\end{array}$ \\
\hline Plan decenal 2015-2025 & 2016 & $\begin{array}{l}\text { Política Nacional de Ciencia, Tecnología e Innovación } \\
2015-2025\end{array}$ \\
\hline
\end{tabular}

Fuente: Elaboración propia del autor (Chávarro, 2018). 
Tras la revisión documental de las fuentes de política científica, se puede afirmar, en primer lugar, que en las leyes que instauran la política científica colombiana (Ley 29 de 1990 y 1286 de 2009), las ideas relevantes corresponden a las aquí denominadas representaciones económica y competitiva de innovación. Así, en el artículo 1 de la Ley 29 de 1990, aparece la idea de incorporar ciencia y tecnología al desarrollo económico, además de que figura como asunto de carácter imperativo cuando se subraya que el Estado «está obligado a incorporar la ciencia y la tecnología a los planes y programas de desarrollo económico» (Ley 29, 1990), y en el mismo sentido, se invoca al sector económico como prioritario. Esto permite mostrar que la representación central reside en la funcionalidad de la ciencia para la productividad, lo que constituye una apropiación efectiva del modelo internacional de innovación, cuyo anclaje institucional se da mediante el recurso al filtro o concurrencia de las representaciones de Estado (Estado como protector de la propiedad intelectual, defensor de los intereses nacionales, vínculo entre los sectores privado y público, etc.) y de los derechos democráticos ciudadanos (Estado como reductor de inequidades de acceso al conocimiento, fomento a la descentralización y empoderamiento regional en el conocimiento, participación de los sectores, etc.), todos estos aspectos propios del entorno institucional y discursivo de la década de 1990 y posteriores, con la entrada en vigencia de la Constitución Política de 1991. De otro lado, en dicho análisis se confirma la inexistencia de indicios relativos a representaciones alternas de innovación; es decir, no figuran asociaciones claras con la innovación social, cultural o ambiental (llámese conocimiento local, tecnologías intermedias, tecnologías sociales, etc.), y relacionada con la anterior, aparece una imagen de desarrollo científico-tecnológico despojada de la idea de riesgo.

Por otra parte, puede afirmarse que en la Ley 1286 de 2009, es donde finalmente aparece el diagrama completo del modelo internacional de innovación. Como ya se ha advertido, el anclaje se produce apelando a la concurrencia de las figuras del Estado, las bondades democráticas y los derechos ciudadanos. Desde el artículo 1, se aclara que el objetivo de esta reside en fortalecer mecanismos o instrumentos necesarios para sacar adelante el objetivo plasmado en la Ley 29 de 1990, «[...] fortalecer el Sistema Nacional de Ciencia y Tecnología y a Colciencias para lograr un modelo productivo sustentado en la ciencia, la tecnología y la innovación», y la idea relevante reside en la función productiva de la ciencia: «[...] para darles valor agregado a los productos y servicios de nuestra economía y propiciar el desarrollo productivo y una nueva industria nacional» (Ley 1286 de 2009, Art. 1).

En segundo lugar, al mirar los múltiples planes de desarrollo (pertenecientes a seis gobiernos de 1990 a 2017), y demás documentos de política científica analizados, en cuanto a las representaciones predominantes en estos se puede ver una tendencia in crescendo respecto de la apropiación del modelo internacional cen- 
trado en la idea de innovación para impulsar la productividad y como ventaja competitiva. A pesar de que los planes de desarrollo, de gobierno a gobierno, tienen variaciones en cuanto a modelos de desarrollo, la orientación por la innovación para la productividad y competitividad se mantiene en todos y crece cada vez más hasta determinar las prácticas, llegando a dejar subordinada la dinámica de ciencia y tecnología a instancias como los consejos de competitividad y a sus agendas específicas, ya en el periodo reciente, desde 2010.

Sin embargo, a diferencia de lo que pasa con las leyes de la política científica, en dichos planes se constata la temprana aparición de lo relativo a la sostenibilidad ambiental, aunque parece estar más motivada por la experiencia de situaciones coyunturales como la aparición de desastres, olas invernales y períodos de sequía, atribuidos al cambio climático. Aunque aparece el uso recurrente de una terminología sobre lo «sostenible y sustentable», no siempre tales adjetivos se vinculan con lo ambiental, lo que se puede interpretar como una forma de anclaje periférico, ya que no llega a afectar el núcleo central de las representaciones económicas y competitivas de innovación.

En cuanto a la presencia de representaciones alternas de innovación, solo aparecen de manera indirecta en algunos de los planes de desarrollo analizados, como la idea de conocimiento local en el informe Visión 2019 — un documento de autoría no necesariamente gubernamental—, en el cual se subraya la importancia del conocimiento ancestral en relación con el potencial biológico y el uso de la biodiversidad. En cuanto a otra representación alterna, como la innovación social, aparece de manera discontinua y esporádica antes de 2010 en varios planes y sus complementos programáticos, pero solo hasta 2011, con la creación del Centro de Innovación Social (CIS), aparece un panorama interesante para el desarrollo de la innovación social, buscando articular la política social y la política ambiental para encontrar soluciones a necesidades locales. Sin embargo, esta mención de la innovación social aparece determinada por la centralidad de las representaciones económicas, puesto que se la asocia más con la responsabilidad social y el emprendimiento social; es decir, dentro de la esfera de los discursos del management de lo social, como complemento de lo económico.

En síntesis, de la revisión de documentos de política científica, se encuentra que, con la Ley 29 de 1990, se da el anclaje del modelo internacional de política científica, y con la idea de innovación, que aparece en la Ley 1286 de 2009, adquiere su grado de objetivación. El isomorfismo institucional se manifiesta en la estructura del sistema de ciencia y tecnología, que luego incluye el nombre de innovación, y que formalmente vincula actores antes inexistentes en el reducido campo científico, con actores casi exclusivamente pertenecientes a las universidades. 


\subsection{Los asuntos controversiales registrados en la prensa}

\subsubsection{Fuentes, selección de notas e identificación de controversias}

Las fuentes utilizadas para el análisis de asuntos controversiales son las notas de prensa provenientes de tres casas periodísticas de referencia en el país. Para dar cuenta de los asuntos controversiales identificados tras la aplicación del índice de controversialidad, se elaboró una forma de clasificación en el que resultan seis asuntos. A continuación, se describen los tres más importantes.

\subsubsection{Análisis de los asuntos controversiales}

\section{Asunto controversial sobre las problemáticas de financiación de la investigación}

En esta controversia sobre el asunto de la financiación, se percibe mejor el perfil de los agentes del campo (representaciones de sujeto), dado que se trata de una discusión sobre los recursos para la ciencia en la que afloran los intereses y valores de los agentes implicados. En el caso de los agentes académico-científicos, su reclamo reside en que los recursos para la investigación $(\mathrm{I}+\mathrm{D})$ se deben aumentar por encima de otros rubros, como formación, infraestructura, apropiación etc. (ACTI):

No es posible callar, hay que decirlo con todas las palabras: la falta de financiamiento de Colciencias es gravísima. De financiadora y conductora de las políticas nacionales de ciencia, ha pasado a ser una agencia de comunicación y eventos. Las regalías están siendo malgastadas, deben ser revisadas urgentemente. Estamos condenando a nuestras mejores iniciativas científicas a un triste final. ¿Asumirá el gobierno su responsabilidad? (Wasserman, 11-08-2013).

Por su parte, para los agentes gubernamentales y tecnocráticos, y también para los agentes políticos regionales, se deben privilegiar los gastos en formación e infraestructura, dado que estas inversiones permiten aumentar los indicadores de capacidades:

Yo pedí que los recursos para formación doctoral fueran de regalías, porque hoy en día el 55\% de recursos de Colciencias se invierten en formación de capital humano. Solo estamos financiando el $6 \%$ de los proyectos que aprobamos. Eso desestimula a la comunidad científica. También pedí que se aprobara un proyecto de fortalecimiento de las ciencias básicas. Son debilidades que se pueden ir corrigiendo (Restrepo, cit. Fog, 20-04-2012).

De otro lado, los agentes del sector burocrático-administrativo —entre los que se contarían las directivas de las instituciones del sistema CTI- y los organismos de control oscilan entre la posición del sector gubernamental tecnocrático y la del sector académico-científico. Se puede inferir de ello que el retiro de dos directores de la secretaría técnica del sistema se deba a la inclinación por los intereses del sector académico-científico: 
Así se dieron las cosas, no me extraña, pues desde hace meses varios gobernadores vienen presionando mi salida porque ven en Colciencias un obstáculo que ha frenado su intención de gastarse la plata de las regalías de Ciencia y Tecnología en lo que ellos quieren ejecutar con entidades que no tienen experiencia en investigación, y no en lo que las regiones y los colombianos necesitan (Arias, cit. revista Semana, 19-07-2014).

En síntesis, con esta controversia sobre financiación, se percibe mejor el mundo de la ciencia nacional como un campo de fuerzas en el que nuevos agentes introducidos a este campo científico por la misma política científica, como la tecnocracia y los políticos, tienen diversas representaciones de su lugar como sujetos en el manejo de la ciencia, y sus representaciones tienen implicaciones en la toma de decisiones, las prioridades y lo que debe hacerse con los recursos del presupuesto. Esto genera una disputa latente, lo que evidencia aún más que las representaciones de ciencia no residen en el aire sino en el cruce de valores e intereses de actores situados en un campo.

\section{Reacción a la politica cientifica y su lugar en planes de desarrollo}

En el asunto controversial sobre la política científica y los planes de desarrollo es donde mejor afloran las ideas de los agentes, cuyos indicios son las imágenes de ciencia, tecnología e innovación que poseen los sujetos que participan en la controversia. El interés primordial de los agentes académico-científicos es la revisión de la política científica, no solo en la forma o el objetivo de institucionalizar la investigación científica, sino en lo sustantivo de la política: en su idea de ciencia como instrumento para el desarrollo.

Los científicos le proponen al presidente conformar una nueva misión de sabios o de notables que pueda dar luces en la formulación de una política de Estado en esta materia. Insisten además en que a pesar de lograr un aumento importante de recursos, gracias a la destinación del 10 por ciento de las regalías para ciencia, tecnología e innovación, la forma como se plantea la asignación de los mismos, al igual que la visión en el alto gobierno sobre la innovación y su manejo como política pública, hace que Colciencias y las instituciones de investigación del país queden relegados, «ocasionando efectos negativos sobre la confianza y la credibilidad en la política científica y tecnológica» (El Tiempo, 18-07-2012).

Por su parte, los agentes gubernamentales tecnocráticos no tienen cuestionamientos a las ideas centrales de la política sino a las procedimentales:

[... la consecuencia en el atraso de Colombia en este sentido, es visible. «El hecho de que las políticas públicas no hayan sido consistentes e importantes y continuas ha llevado a que Colombia no se destaque en el concierto de países del mundo ni de Latinoamérica. Otras naciones de igual nivel de desarrollo que el nuestro presentan indicadores y avances más contundentes» (Perfetti, cit. Morales, 05-04-2010). 
El aporte indudable de analizar este asunto controversial reside en la consideración conflictiva de las representaciones de ciencia en la política científica y los planes de desarrollo, de lo que se entiende allí por ciencia y de su lugar en el desarrollo. Al cuestionar la innovación, los representantes de la comunidad científica actúan desde sus posiciones dentro de un campo o mundo social en el que se han desempeñado realizando investigación científica, por lo que no comparten la reducción funcional de la ciencia a la productividad.

\section{Ciencia básica vs ciencia aplicada}

En este asunto controversial es donde mejor se percibe el cuadro completo de las representaciones de sujeto, objeto y proyecto de los agentes y cómo esto se articula con la existencia de un campo. Para los agentes del sector académico-científico, la ciencia se representa como investigación desligada de funcionalidades específicas y de búsqueda de utilidades inmediatas:

¿Cuánta física del estado sólido y cuánta química de materiales hay detrás de una pantalla táctil? ¿De dónde salió la tecnología de transmisión inalámbrica de la información? [...] Es difícil profetizar, pero sí se puede decir con seguridad que las innovaciones tecnológicas revolucionarias surgirán en aquellos países que tienen una sólida capacidad científica (Wasserman, 28-05-2015).

Por otro lado, para los agentes de la tecnocracia, el objeto ciencia significa realmente ciencia aplicada, en lo posible de funcionalidad económica, y el proyecto tiene que ver con la orientación de la investigación a la generación de innovaciones productivas. Los otros sujetos, agentes o sectores tienen proyectos con la ciencia seguramente más ligados a la búsqueda de beneficios en términos de un capital propio de sus campos, como los resultados a corto plazo para los agentes políticos o la burocracia tradicional.

En conclusión, la discusión entre ciencia básica y aplicada apunta a las representaciones de objeto de la política científica, dado que muestra cómo la ciencia representa investigación básica para los agentes académico-científicos —un tipo de investigación que da autonomía al mundo social de la ciencia-, mientras que para los sectores político-burocráticos y los de tipo tecnocrático representa aplicaciones útiles en el sentido de mercado, y en términos de campo, la representación de la ciencia para los agentes diversos que pone en contacto la política científica se torna en objeto controversial, dado que dicha política pone en disputa valores como la autonomía y la utilidad, es decir, dos valores claves que diferencian a los denominados modelo lineal y modelo internacional de innovación, o si se quiere, a los regímenes disciplinario y utilitario. 


\subsection{Las percepciones de los entrevistados}

De los múltiples interrogantes planteados a los agentes del campo científico entrevistados, se consigna a continuación los relativos a la situación de la ciencia en el país, y la percepción de la política científica en sus diversos aspectos.

\subsubsection{Percepciones sobre la situación de la ciencia en el país}

Desde la perspectiva de los agentes investigadores, la situación de la ciencia en un país como Colombia es «regular», propia de un país en vías de desarrollo, y el panorama que ofrece no es muy alentador:

[...] es muy difícil, digamos, pensar que vamos a tener una... una ciencia y una investigación ideales, digamos así como... como uno lo puede encontrar en un país de alto nivel de desarrollo o eso... muy difícil, o sea, en nuestro tiempo, no se da ni de fundas, pues (GB, entrevista personal, 2016).

La situación de la ciencia en el país desde la perspectiva de los agentes vinculados con la gestión, evaluación y apropiación social de la ciencia es diversa, pero coinciden con los investigadores en que la situación es regular debido a los problemas derivados de la implementación de la política científica:

La situación de la ciencia en Colombia no está mala, pero está muy lejos de ser lo que debería, con el talento y los recursos físicos y naturales que tenemos y las oportunidades que se nos han abierto [...] (NEH, entrevista personal, 2016).

Las diferencias de percepción de alguna manera tienen que ver con sus posiciones en el campo, ya que hacer parte de la gestión implica compartir de alguna manera la política científica implementada, pero ser investigador permite cuestionarla dados los cambios que introduce para el ejercicio de la investigación y la factibilidad de alcanzar recursos para ello.

\subsubsection{Percepciones puntuales de la política científica colombiana}

$\mathrm{Al}$ introducir el interrogante sobre la percepción de la política científica vigente, los investigadores la perciben como una expresión del modelo utilitarista de ciencia, que privilegia solo el último momento de la cadena de conocimiento:

Pues mirá, yo creo que hay un gran problema y es la concepción de la política... eh... científica en Colombia. Yo creo que la concepción es una concepción muy utilitarista eh... es una concepción, además eh... que es muy ingenua con respecto a lo que es y significa la ciencia en un país como Colombia... eh... ingenua en el peor sentido de la palabra eh [...] Considero profundamente inadecuada para lo que podría ser la dinámica de la ciencia en Colombia... (ER, entrevista personal, 2016).

De otro lado, en la perspectiva de los agentes de gestión, evaluación y apropiación social, se destaca el desbalance de la política científica entre objetivos e 
instrumentos y la imposición de un sistema por decreto dada la débil institucionalidad, entre otros aspectos:

Colciencias es recesivo por decirlo de alguna manera, es como un gen recesivo dentro del sistema [...], entonces no hay una institucionalidad que le preste una visión al país y que negocie una visión ante el problema. Ese es, dijéramos, un poco el panorama. (HJ, entrevista personal, 2016).

En otras palabras, para los investigadores la percepción crítica de la política científica tiene que ver con las ideas centrales provenientes del modelo de innovación y los instrumentos de financiación que limitan las posibilidades de hacer investigación básica. Por el contrario, para los gestores, la percepción de dicha política tiene que ver solo con la falta de racionalidad de los instrumentos puestos en juego, no con sus ideas centrales.

Respecto del interrogante sobre la percepción o conocimiento de los asuntos controversiales de la política científica registrados en la prensa, en las respuestas de los entrevistados se pudo constatar que los investigadores comparten las discusiones ventiladas en los medios de opinión pública, y que tienen que ver con los debates sobre la financiación, la medición de grupos y publicaciones, el retorno de cerebros, etc., todos conocidos por los entrevistados. Su percepción agrega detalles sobre ellos, e incluso algunos sugieren que hay discusiones que no han alcanzado registro en la prensa, como la falta de coordinación del sistema, la asimetría de las regiones en capacidades, los problemas de las becas de doctorado, y la asimetría en la asignación de recursos según el ranking de los investigadores.

Por su parte, para los actores vinculados con la gestión de la ciencia, lo que indica el surgimiento de asuntos controversiales tiene que ver con otros problemas relacionados, como la inconsistencia dinámica de las políticas públicas, la falta de compromiso del sector privado con la innovación, el cortoplacismo provinciano en la asignación de recursos a las prioridades de ciencia y tecnología, el funcionamiento de los rankings como normativos para la asignación de recursos, y la inexistencia de fuentes de empleo para los doctorandos que regresan al país. Este conjunto de detalles, aportados desde la perspectiva de los actores que se mueven en la gestión de la ciencia, arroja otra explicación a las reacciones a la política científica, pero desde la racionalidad del modelo que tiene la política científica y sus problemas formales o instrumentales de aplicación.

En cuanto al interrogante sobre el principal problema de la política científica, la mayoría de entrevistados coincide en lo relativo a la financiación y la coordinación del sistema, que serían problemas propios de instrumentos:

El problema clave es un problema de falta de política sobre la ciencia y falta de financiación. Se refleja en que no hay unas reglas claras, una valoración clara y efectiva del quehacer científico... (LFG, entrevista personal, 2016). 
Curiosamente, el investigador en ciencias sociales entrevistado destaca como problema la forma en que se privatiza el conocimiento, financiado con fondos públicos, a través de las publicaciones indexadas y bases de datos internacionales:

Cuando, por ejemplo, Colciencias, con Publindex, le apuesta a que son ISI y Scopus los paradigmas de medición del asunto, lo que está haciendo es... escúchame esto, lo que está haciendo es privatizando resultados de conocimientos, producidos muchos de ellos con recursos estatales públicos... (ER, entrevista personal, 2016).

Respecto de otro interrogante —esta vez sobre la situación de las regiones-, la percepción de los agentes científicos no es vista con mucho optimismo. En particular, se afirma que no es suficiente que una región posea capacidades para el ejercicio de la ciencia, ya que se dan disparidades:

[...] Eh, yo creo que es poco lo que se puede hacer desde lo local... para que pueda florecer actividad científica relevante por la historia de centralismo en Bogotá, pero también por la rapiña que existe en esos escenarios... Yo soy pesimista con respecto al asunto (ER, entrevista personal, 2016).

De otro lado, para los agentes vinculados más con la gestión de la ciencia, la disparidad regional se traduce en que algunas regiones con buenas capacidades agencian políticas locales y sectorizadas, pero otras regiones no cuentan ni con centros de educación superior o universidades de tradición científica, y este déficit genera más inquietudes que respuestas.

\section{DisCUSIÓN Y CONCLUSIONES}

Como se expresó al inicio de este trabajo, las ideas centrales de la política científica colombiana provienen de la difusión de un modelo internacional centrado en la innovación, gestado en organizaciones internacionales y socializado en el mundo. La apropiación de este modelo internacional en la política científica colombiana ha sido gradual, desde su inicio en la década de 1990 hasta la actualidad. Para mirar este proceso, aquí se ha adoptado el enfoque cognitivo de las representaciones.

El proceso de anclaje de estas ideas centrales del modelo internacional llevó casi dos décadas hasta alcanzar su objetivación (de 1990 a 2009). Tras la objetivación del modelo en la figura de la innovación, la política científica logró no solo imponer la funcionalidad productiva de la ciencia, sino llevar cada vez el sistema de investigación a una dependencia fuerte de la política de competitividad, aspecto que se evidenció cuando la política científica empezó a depender de la Agenda de Competitividad.

La segunda proposición planteada en este trabajo tiene que ver con la apropiación conflictiva del modelo mencionado. Para dar luz a este aspecto se acudió al 
enfoque de las controversias, y su referente empírico se buscó en las reacciones a la política científica registradas en la prensa de referencia del país. El análisis realizado permitió mostrar la existencia de un conjunto de controversias en la prensa, en torno a aspectos como la financiación de la investigación científica, las ideas centrales de la política científica, programas adoptados como el «retorno de cerebros», la discusión entre ciencia básica y aplicada, las formas de medición de los grupos de investigación y las publicaciones, y la discusión del lugar que año tras año ocupa el país en los ranking de innovación y competitividad en el mundo.

Para mirar estas reacciones, no bastaba considerarlas un referente de la apropiación conflictiva del modelo de política científica, sino tratar de entender su sentido para los agentes del mundo social de la ciencia. Como ya se ha mostrado, las ideas tienen que ver con las representaciones de ciencia, tecnología e innovación que tienen los agentes que reaccionan en la prensa, que, en este caso, difieren en gran parte de las representaciones centrales del modelo de política científica. En otras palabras, la ciencia para los científicos corresponde al primer momento de la innovación, ya que sin investigación básica no se puede tener descubrimientos que permitan innovaciones radicales. Sin embargo, esto rińe con la idea central de los agentes de la tecnocracia gubernamental de acuerdo con la cual la investigación debe ser aplicada para generar innovaciones cuyos productos tengan demanda en el mercado y generen valor. La defensa de estas representaciones de ciencia, en términos de campo, muestra que sin financiación de investigación básica se perjudican los intereses de los científicos, por lo que se evidencia que la política científica y la creación de un sistema con actores diferentes de los científicos pone presente una crisis del campo o mundo social de la ciencia, y ello explica la aparición de asuntos controversiales.

Lo anterior se corrobora empíricamente tras el análisis de entrevistas a agentes del mundo científico acerca de su percepción de la política científica. Para estos agentes, la situación de la ciencia en el país es regular tirando a mala y la política científica es percibida como equivocada, sobre todo por los investigadores, quienes cuestionan el fondo mismo de aquella, mientras que los gestores solo ven como problemáticos los temas de aplicación de programas y la falta de racionalidad para llevar a cabo los objetivos, y califican las críticas a la política como retórica que esconde intereses. Sin embargo, lo que muestra el estudio de percepción es la confirmación de la apropiación conflictiva de dicha política, y la crisis en que ha sumido al campo nacional de la ciencia.

En síntesis, con la implementación de la política científica colombiana, se crea el sistema de ciencia, tecnología e innovación, que permite el ingreso de nuevos agentes al mundo de la ciencia que buscan las posiciones claves del campo desde unas ideas de ciencia propias del modelo internacional de innovación. A la llegada de más agentes al campo, se agrega una fragmentación de la identidad de los agentes científicos, entre científicos naturales y sociales. Sin embargo, la lucha 
de los agentes científicos obtiene algo de lo buscado en las reivindicaciones. Por ejemplo, el sistema admite ya financiar por igual a investigadores de universidades que a investigadores de centros de investigación y centros tecnológicos. Asimismo, aspectos como la estrategia de spin-off y la creación de start-up ya es posible para los investigadores de las universidades públicas. Además, la secretaría técnica acaba de convertirse en Ministerio de Ciencia y Tecnología. Sin embargo, la mayoría de los aspectos seńalados en los asuntos controversiales sigue dando vueltas en la opinión, y se muestra que desde la tecnocracia, lo primero que se sacrifica es el presupuesto de la ciencia ante las dificultades de coyuntura, como la caída del precio del petróleo o la construcción de vías de cuarta generación, lo que muestra una brecha entre las representaciones de ciencia del modelo importado y las representaciones sociales de la ciencia entre los agentes de la ciencia y la política en la sociedad colombiana.

En términos de discusión, queda la inquietud de que el abordaje de las reacciones a la política científica desde un enfoque de campo se pueda desarrollar mejor, considerando que la ciencia no necesariamente forma parte de un microcosmos social nacional, sino que existen relaciones internacionales entre los campos científicos nacionales, así como una dimensión de la ciencia como campo global. El hecho de que este problema esté presente en muchos países, como expresan Pestre (2005) y el mismo Bourdieu (2003), muestra que una consideración del mundo social de la ciencia como campo global pudiera tener sentido (Syvatera, 2016), así como una consideración de la ciencia como subcampos o regímenes múltiples que se relacionan, interactúan y coexisten, como han señalado Marcovitch y Shinn (2012) al identificar la coexistencia del régimen disciplinar y el régimen utilitarista de la ciencia. Aspectos como lo relativo a la marcha mundial por la ciencia permiten pensar que es posible examinar la dimensión global del campo científico, y la coexistencia de las universidades con los centros de investigación, los centros tecnológicos y las iniciativas privadas con la ciencia, y así inferir que la situación actual de la política científica tiene que ver con que pone en contacto varios regímenes de la ciencia que han funcionado independientemente, por lo que ha resultado difícil meterlos en el mismo sistema. Ello ayuda a entender la diferencia de representaciones de ciencia, de percepciones de la política científica, sus controversias y la dificultad de asimilarlos dentro de un esquema, como ha intentado hacer la política en cuestión con la creación del sistema. Todos estos elementos ayudan a entender el porqué del malestar con la innovación.

De alguna manera, también, lo expresado en este trabajo contribuye modestamente a mirar alternativas. En primer lugar, haciendo evidente que, más allá del modelo lineal de autonomía y el modelo internacional de innovación de la política científica — denominados como Modo 1 y Modo 2 (Gibbons et al., 1994)—, se puede pensar en un modelo ciudadano de política científica (Mitcham y Bri- 
ggle, 2007) que pueda realmente involucrar actores con una cultura política cívica (Elzinga y Jamison, 1996), que contribuya a generar un sentido de relevancia desde lo público (Vasen, 2011), y donde la innovación no se reduzca a su representación económica y competitiva, sino que asimile en su núcleo la innovación social y ambiental, como plantea quien aquí escribe, sin cuyos pilares resulta difícil pensar las significaciones de la ciencia en el siglo XXI. Desde luego, sin olvidar que el mundo de la ciencia es un campo de fuerzas (Bourdieu, 2000), por lo que el conflicto latente de tomas de posición respecto de las prioridades de la ciencia no debe verse como algo patológico sino como una oportunidad de creación, es decir, una de las mejores significaciones que pueda atribuirse a la palabra innovación.

\section{REFERENCIAS}

Albornoz, M. (2009). Desarrollo y políticas públicas en ciencia y tecnología en América Latina. Revista de investigaciones politicas y sociológicas (RIPS), 8(1), 65-75.

Albornoz, M. (2007). Los problemas de la ciencia y el poder. Revista Iberoamericana de Ciencia, Tecnología y Sociedad, 3(8), 47-65.

Bauer, M. \& Gaskell, G. (1999). Toward a paradigm for research on social representations. Journal for the Theory of Social Behaviour, 29(2), 163-182.

Bernal, J. D. (1938). The social function of science. Recuperado de https:/www.marxists.org/ archive//bernal/works/1930s/socialscience.htm

Bourdieu, P. (2003). El oficio de científico. Barcelona: Anagrama.

Bourdieu, P. (2000). El campo cientifico. Usos sociales de la ciencia. Buenos Aires: Nueva Visión.

Brante, T. \& Elzinga, A. (1990). Towards a theory of scientific controversies. Science Studies, 2, 33-46.

Bush, V. (1999). Ciencia, la frontera sin fin. Redes. Revista de Estudios Sociales de la Ciencia, 14, $1-30$.

Casas Guerrero, R. et al. (2013). Políticas de ciencia, tecnología e innovación en América Latina: entrela competitividad yla inclusión social. Conferencia internacionalLALICS. Recuperado de http://www.redesist.ie.ufrj.br/lalics/papers/115_politicas_de_ciencia_tecnologia_e_ innovacion_en_america_latina_entre_la_competitividad_y_la_inclusion_social.pdf

Chávarro, L. A. (2018). El malestar con la innovación. Una aproximación a la dinámica controversial de la politica científica colombiana 1990-2017 (Tesis de doctorado en Sociología). Lima: PUCP.

Collins, H. \& Pinch, T. (1996). El golem. Lo que todos deberíamos saber acerca de la ciencia. Barcelona: Crítica.

Departamento Nacional de Planeación DPN (1990). Ciencia y tecnología. En La revolución pacifica 1990-1994. Recuperado de https://colaboracion.dnp.gov.co/cdt/pnd/gaviria_ estrategias_del_plan5.pdf

Departamento Nacional de Planeación DPN (1994). Competitividad para la internacionalización. En El salto social 1994-1998. Recuperado de https://colaboracion.dnp.gov.co/cdt/ pnd/samper_competitividad_internacionalizacion.pdf

Departamento Nacional de Planeación DPN (1998). Exportaciones como motor de crecimiento. En Cambio para construir la paz 1998-2002. Recuperado de https://colaboracion.dnp. gov.co/cdt/pnd/pastrana2_exportaciones_motor.pdf 
Departamento Nacional de Planeación DPN (2002). Hacia un estado comunitario 2002-2006. Recuperado de https://colaboracion.dnp.gov.co/cdt/pnd/pnd.pdf

Departamento Nacional de Planeación DPN (2006). Estado comunitario: desarrollo para todos 2006-2010. Recuperado de https://colaboracion.dnp.gov.co/cdt/pnd/pnd_tomo_2.pdf

Departamento Nacional de Planeación DPN (2010). Prosperidad para todos 2010-1014. Recuperado de https://sinergia.dnp.gov.co/sismeg/archivos/pnd2010-2014\%20tomo\%20 i\%20cd.pdf

Departamento Nacional de Planeación DPN (2014). Todos por un nuevo país 2014-2018. Recuperado de https://colaboracion.dnp.gov.co/cdt/prensa/bases\%20plan\%20nacional\%20 de\%20desarrollo\%202014-2018.pdf

Di Maggio, P. \& Powell, W. (1999). Retorno a la jaula de hierro: el isomorfismo institucional y la racionalidad colectiva en los campos organizacionales. En El nuevo institucionalismo en el análisis organizacional (pp. 104-125). México: FCE.

Díaz Moreno, N. \& Jiménez, M. R. (2012). Las controversias socio-científicas: temáticas e importancia para la educación científica. Revista Eureka sobre Enseñanza y Divulgación de las Ciencias, 9(1), 54-70. Recuperado de http://sciencepolicy.colorado.edu/students/ envs_5100/polanyi_1967.pdf

Dimopoulos, K. \& Koulaidis, V. (2002). The socio-epistemic constitution of science and technology in the Greek press: an analysis of its presentation. Public Understanding of Science, $11,225-241$.

Edgerton, D. (2004). The linear model' did not exist: reflections on the history and historiography of science and research in industry in the twentieth century. En K. Grandin y N. Wormbs (eds.), The science-industry nexus: history, policy, implications. Nueva York: Watson.

El Tiempo (18 de julio de 2012). Científicos le piden a santos claridad sobre política de ciencia. El Tiempo. Recuperado de http://www.eltiempo.com/archivo/documento/CMS-12042264

Elzinga, A., Jamison, A. (1996). El cambio de las agendas políticas en ciencia y tecnología. Zona Abierta, 75-76, 2- 22.

Etzkowitz, H. \& Leydesdorff, L. (2000). The dynamics of innovation: from national systems and "Mode 2" to a Triple Helix of University-Industry-Government Relations. Research Policy, 29, 109-123.

Finnemore, M. (1993). International organizations as teachers of norms: The United Nations educational, scientific, and cultural organization and science policy. International Organization, 47(4), 565- 597.

Fog, L. (20 de abril de 2012). Director de Colciencias habla sobre regalías. El Espectador. Recuperado de http://www.elespectador.com/noticias/actualidad/vivir/director-decolciencias-habla-sobre-regalias-articulo-340006

Forero, C. \& Villaveces J. L. (2002). Objetivos, marco conceptual y metodología. En C. Forero, J. L. Villaveces et al. La evaluación de programas de investigación y su impacto en la sociedad colombiana. Bogotá: Colciencias.

Garay, L. J. et al (1998). Colombia: estructura industrial e internacionalización 1967-1996. Bogotá: DPN-Colciencias.

Gibbons, M., Limoges, C., Nowotny, H. Trow, M., Scott, P., Schwartzman, S. (1994). La nueva producción del conocimiento. Barcelona: Pomares.

Gómez, Y.J. (2005). Política científica colombiana y bibliometría: usos. Nómadas, 22, 241-254.

Guzmán Aguilera, C. L. (2014). Política científica colombiana. ¿Conocimiento para todos? el derecho a la igualdad. Anfora, 21(36), 17-39. 
Herrera, A. (1995). Los determinantes sociales de la política científica en América Latina. Política científica explícita y política científica implícita. Redes, 5, 115-126.

Hernández Aguirre, M. I. (2011). Análisis comparativo del discurso de las políticas públicas de ciencia, tecnología e innovación en Colombia. Hologramática, 2(14), 137-167.

Hoyos, G. (1991). Elementos filosóficos para la comprensión de una política de ciencia y tecnología. En A. A. V.V. Colombia, el despertar de la modernidad (pp. 396-447), Bogotá: Norma.

Jaramillo, H. (2007). Colombia: evolución, contexto y resultados de las políticas de ciencia, tecnología e innovación. En J. Sebastián (ed.), Claves del desarrollo científico y tecnológico de América Latina (pp. 301-329). Madrid: Fundación Carolina-Siglo XXI.

Marcovich, A., Shinn, T. (2012). Regimes of science production and diffusion: towards a transverse organization of knowledge. Scientic Zudia, 10, 33-64.

Malaver, F. \& Vargas, M. (2005). Políticas y avances en la ciencia, la tecnología y la innovación en Colombia. Cuadernos de Administración, 18(30), 39-78.

Meyer, J. W., Boli, J., Thomas, G., \& Ramirez, F. O. (1997). World society and the nation-state. American Journal of Sociology, 103(1), 144-181. https://doi.org/10.1086/231174

Mitcham, C., Briggle, A. (2007). Ciencia y política: perspectiva histórica y modelos alternativos. Revista CTS, 3(8), 143-158.

Morales, M. (5 de abril de 2010). Pobreza de Colombia en apoyo a la ciencia. El Tiempo. Recuperado de http://www.eltiempo.com/archivo/documento/MAM-3906915

OCDE (2014). Estudio de la OCDE de las políticas de innovación de Colombia. Recuperado de https://www.oecd.org/sti/inno/colombia-innovation-review-assessment-and-recommendations- spanish.pdf

Pestre, D. (2005). Ciencia, dinero y política. Buenos Aires: Nueva Visión.

Polanyi, M. (1962). The republic of science. Minerva 1, 54-74. Recuperado de http://sciencepolicy.colorado.edu/students/envs_5100/polanyi_1967.pdf

Revista Semana (19 de julio de 2014). Varios querían mi cabeza. Revista Semana. Recuperado de http://www.semana.com/nacion/articulo/varios-querian-mi-cabeza/396177-3

Rosenberg, N. (1994). Incertidumbre y cambio tecnológico. Revista de Historia Industrial, 6, 11-30. Sampat, B. (2007). Política científica y tecnológica de Estados Unidos: reseña histórica e implicancias para los países en desarrollo. Conferencia en la CEPAL, Santiago de Chile, 6 y 7 de diciembre. Recuperado de https://www.cepal.org/iyd/noticias/paginas/4/31424/ sampat.pdf

Sagasti, F. (2013). Ciencia, tecnología, innovación. Políticas para América Latina. Lima: FCE.

Schumacher, E. F. (1983). Lo pequeño es hermoso. Barcelona: Orbis.

Syväterä, J. (2016) Creation and domestication of global policy trends. Tampere - Finlandia: Universidad de Tampere.

Turriago, A. y G. Hernández (2011). Análisis de capacidades y evolución del sistema nacional de ciencia, tecnología e innovación en Colombia. Cuadernos Latinoamericanos de Administración, 7(12), 49-60.

Vasen, F. (2011). Los sentidos de relevancia de la política científica. Revista CTS, 7(19), 11- 46. Vessuri, H. (1994). La ciencia en América Latina en el siglo XX. Redes, 1(2), 41-76.

Wasserman, M. (28 de mayo de 2015). La punta del iceberg y la casita en el aire. El Tiempo. Recuperado de http://www.eltiempo.com/opinion/columnistas/la-punta-del-iceberg-yla-casita-en-el-aire-moises-wasserman-columnista-el-tiempo/15847295

Wasserman, M. (11 de agosto de 2013). Ciencia cenicienta. El Tiempo. Recuperado de http:// www.eltiempo.com/archivo/documento/CMS-12985790 